\title{
Polimorfismos genéticos asociados a la inmunidad innata y la susceptibilidad genética a la tuberculosis
}

\author{
NANCY P. MAULÉN* y LUCÍA CIFUENTES O.**
}

\author{
Genetic polymorphisms associated with innate immunity and to genetic susceptibility \\ to tuberculosis
}

Tuberculosis is a global health problem exacerbated by the absence of an effective vaccine and emergence of extensive antibiotic-resistant strains. Innate immunity is key to tuberculosis susceptibility, since it is associated with genetic polymorphisms in TLRs (Toll Like Receptors), VDR (Vitamin D Receptor), INF- $\gamma, T N F-\alpha$, among others. Recently, also to nine Mendelian Susceptibility Mycobacterial Diseases (MSMD) -causing genes, including autosomal and X-linked genes. After decades of successful management, Chile reported maintenance of mortality and increase in tuberculosis under 15 years and multidrug resistant cases incidence. Moreover, incidence is higher in the North and the Center, where Metropolitan Region showed a sustained increment of the Latin American migrant population. Consequently, the high incidence persistence in such geographic areas could be associated with populations carrying SNPs genetic polymorphisms types and/or MSMD that confer genetic susceptibility to tuberculosis and/or BCG dissemination and other intramacrophagic pathogens, similar to that described in certain populations in Europe, Asia, Africa and America. Corollary, we propose to consider genetic predisposition of the current population, at the time of designing national public health policies to eradicate tuberculosis.

Key words: Genetic predisposition to disease; tuberculosis; mycobacterium; polymorphism, single nucleotide; immunity, innate.

\section{Resumen}

La tuberculosis es un problema de salud mundial exacerbado por la ausencia de una vacuna eficaz y la emergencia de cepas multidrogo resistentes. La inmunidad innata, clave en la susceptibilidad a tuberculosis, está asociada a polimorfismos genéticos en TLRs (Toll Like Receptors), VDR (Vitamin D Receptor), INF- $\gamma$, TNF- $\alpha$, entre otros. Recientemente, también a nueve genes causantes de susceptibilidad mendeliana a enfermedades micobacterianas (MSMD), incluyendo genes autosómicos y ligados al cromosoma X. Después de décadas de exitoso manejo, Chile reportó mantención de la mortalidad, aumento en la incidencia de tuberculosis en todas sus formas y casos multidrogo resistentes. La incidencia es mayor en Norte y Centro, donde la Región Metropolitana mostró incremento de población migrante latinoamericana. Consecuentemente, la alta persistencia de la incidencia en tales zonas geográficas, podría asociarse a poblaciones portadoras de polimorfismos de un solo nucleótido (SNP)s y/o MSMD, confiriendo susceptibilidad genética a tuberculosis y/o a BCG diseminada y otros patógenos intramacrofágicos, similar a lo descrito en poblaciones de Europa, Asia, Africa y América. En conclusión, proponemos considerar la predisposición genética de la población actual, al momento de diseñar políticas nacionales de salud pública para erradicar la tuberculosis.

Palabras clave: Predisposición genética a enfermedad; tuberculosis; micobacteria; polimorfismo de un solo nucleótido; inmunidad, innata.

Financiamiento: Sin apoyo financiero.

* Universidad de los Andes. Escuela de Nutrición y Dietética. Facultad de Medicina. Santiago, Chile.

** Programa de Genética Humana, ICBM. Facultad de Medicina. Universidad de Chile. Santiago, Chile. 


\section{Introducción}

La tuberculosis (TB), causada por Mycobacterium tuberculosis (Mtb), es la novena causa mundial de muerte y la primera por enfermedades infecciosas, incluso por sobre el VIH/SIDA. La TB es frecuente en grupos sociales desposeídos, por ello un problema de salud global exacerbado por la falta de una vacuna eficaz y la emergencia de cepas multirresistentes y extensivamente resistentes a drogas (MDR-TB y XDR-TB). El 2016, 10,4 millones de personas contrajeron la TB, donde $1,0 \mathrm{M}$ correspondió a niños $(10 \%)$ y 1,2 $\mathrm{M}$ a VIH-positivos (11\%). La muerte por TB fue de 1,3 millones en personas VIH-negativas y de 374.000 en VIH-positivas. El mayor número de casos incidentes correspondieron a Asia Sudoriental, África y Pacífico Occidental; las proporciones fueron menores en las regiones del Mediterráneo Oriental, Europa y las Américas (Tabla 1). Estos datos corresponden a 202 países y territorios que dan cuenta de más del $99 \%$ de la población mundial y casos de TB. El 2015, Naciones Unidas adoptó los "Objetivos de Desarrollo Sostenible" (ODS) 2016-2030, siendo uno de éstos terminar con la epidemia de TB, objetivo común a la estrategia "Stop TB" de la OMS, ambas proponen lograr la reducción del $90 \%$ de las muertes por TB y el $80 \%$ de la tasa de incidencia ${ }^{1}$.

En Chile, la incidencia de la TB es baja y la endemia evolucionó por años hacia la declinación en la morbilidad. Sin embargo, después de décadas de exitoso manejo de Salud Pública, en los últimos cinco años, se evidenció la mantención de la mortalidad y el aumento de la incidencia a $[\sim 14 / 100.000]$, valor semejante al de la población mundial (Tabla 2). En 2016, la morbilidad total de TB en todas sus formas

Tabla 1. Carga Global de Tuberculosis. OMS, 2017

\begin{tabular}{|lc|}
\hline Región OMS 2016 & $\begin{array}{c}\text { Carga [\%] } \\
\text { Tuberculosis* }\end{array}$ \\
\hline África & 25 \\
Américas & 3 \\
Mediterráneo Oriental & 7 \\
\hline Europa & 3 \\
Asia Sudoriental & 45 \\
Pacífico Occidental & 17 \\
\hline
\end{tabular}

*La mayor parte del número estimado de casos incidentes (casos nuevos más recaídas) en 2016 correspondió a las Regiones de Asia Sudoriental (45\%), África (25\%) y Pacífico Occidental (17\%); las proporciones fueron menores en las Regiones del Mediterráneo Oriental (7\%), Europa (3\%) y las Américas (3\%).
(TBTF: casos nuevos más recaídas) fue de 14 por 100.000 habitantes y la incidencia de 13,2 por 100.000 habitantes, igual a la tasa del año 2015. La incidencia TBTF fue de [40,0/100.000] en la región de Tarapacá (I), es decir, una incidencia $\sim 2$ veces mayor que en cualquier otra región del país, seguida por la regiones de Arica y Parinacota $(\mathrm{XV})$ con $[26,7 / 100.000]$ y Atacama (III) con [16,1/100.000] (Tabla 3).

Tabla 2.-Incidencia de tuberculosis en Chile. Período 2006 a 2016

\begin{tabular}{|cc|}
\hline Año & $\begin{array}{c}\text { Incidencia } \\
\text { [casos nuevos/100.000 habitantes] }\end{array}$ \\
\hline 2006 & 15,7 \\
\hline 2007 & 14,9 \\
\hline 2008 & 14,7 \\
\hline 2009 & 14,6 \\
\hline 2010 & 14,6 \\
\hline 2011 & 14,5 \\
\hline 2012 & 14,0 \\
\hline 2013 & 13,5 \\
\hline 2014 & 13,4 \\
\hline 2015 & 14,2 \\
\hline 2016 & 14,0 \\
\hline
\end{tabular}

Fuente: Programa de Control de la Tuberculosis. Ministerio de Salud. Chile.

Tabla 3. Incidencia de Tuberculosis en todas sus formas por regiones de Chile en el período 2006 a 2016

\begin{tabular}{|cc|}
\hline Región & $\begin{array}{c}\text { Incidencia } \\
\text { [casos nuevos/100.000 habitantes] }\end{array}$ \\
\hline I & 40,0 \\
\hline II & 15,7 \\
\hline III & 16,1 \\
IV & 11,5 \\
\hline V & 9,3 \\
VI & 8,2 \\
\hline VII & 9,3 \\
\hline VIII & 12,7 \\
\hline IX & 10,5 \\
\hline X & 15,9 \\
\hline XI & 7,3 \\
\hline XII & 11,5 \\
\hline XIII & 15,3 \\
\hline XIV & 13,5 \\
\hline XV & 26,7 \\
\hline
\end{tabular}

Fuente: Programa de Control de la Tuberculosis. Ministerio de Salud. Chile. 
Respecto a los grupos vulnerables el 21,6\% correspondió a adultos mayores, $12 \%$ a extranjeros y $8,7 \%$ a coinfectados con VIH. Asimismo, tanto la coinfección TB-VIH como los casos de TB extrapulmonar aumentaron y la cobertura de BCG disminuyó. Entre los casos nuevos de tuberculosis 13 fueron TB-MDR (multidrogo resistentes), cantidad bajísima respecto de los 4.500 casos reportados en la región de Las Américas el año 2016 ${ }^{1}$. También, los estudios de contactos en el hogar y seguimiento fueron insuficientes, hechos de preocupación porque estos son el grupo de mayor riesgo y probablemente los futuros casos de TB. Dentro de los estudios de contacto se diagnosticaron 13 casos de tuberculosis activa en niños menores de 15 años. Así, el $80 \%$ de los casos son de tuberculosis pulmonar y alrededor del $90 \%$ son confirmados bacteriológicamente, donde la incidencia se ha incrementado a 9,2 por 100.000 habitantes, siendo el 2013 de $8,1^{2}$. Por otra parte, se mantiene la extrema variabilidad entre las 15 regiones del país, siendo una característica epidemiológica relevante, hecho a considerar al momento de diseñar una estrategia nacional de control y prevención de la $\mathrm{TB}^{1}$.

En Chile, no se han reportado estudios acerca de la susceptibilidad genética a la tuberculosis de la población, tampoco se ha identificado polimorfimos genéticos asociados a los distintos fenotipos de la enfermedad y/o en pacientes coinfectados con VIH, según las bases de datos PubMed, ScienceDirect y Scielo, en búsqueda realizada el 5 de agosto de 2018 con las palabras clave "tuberculosis, fenotipo, TB VIH, Chile". No obstante, existen diferencias entre los perfiles de los pacientes que padecen tuberculosis en las distintas zonas geográficas del país, donde los pueblos indígenas Mapuche y Aymará son el principal grupo de riesgo para las regiones de $\mathrm{La}$ Araucanía y de Arica-Parinacota ${ }^{1}$.

En conclusión, el Programa Nacional de Control de la tuberculosis ha mostrado dificultades en varios aspectos de sus operaciones, con el consiguiente riesgo de progresar hacia una reversión epidemiológica en el país. En este contexto, el Ministerio de Salud, ha debido posponer los plazos comprometidos para lograr la meta de la Estrategia Nacional de Salud "alcanzar la eliminación de la tuberculosis como problema de Salud Pública"'. Una primera medida que permitiría acercarse a la meta señalada es mantener y reforzar las medidas epidemiológicas del Programa Nacional de Control de la Tuberculosis que han sido exitosas y agregar otras estrategias adicionales.

\section{M. tuberculosis y su adaptación a poblaciones humanas específicas}

Los mecanismos de patogenicidad de Mtb, le permiten invadir los macrófagos alveolares de su hospedero, interferir con la acidificación del fagosoma, tanto como mediar su replicación intracelular y diseminación linfohematógena. Los macrófagos humanos tienen funciones efectoras dirigidas a restringir la proliferación intracelular de las micobacterias, tales como la autofagia y péptidos antimicrobianos ${ }^{4}$. Sin embargo, en alrededor del 10\% de los individuos, Mtb evade los mecanismos de defensa del hospedero y establece una enfermedad activa (TB pulmonar). En el $90 \%$ de las personas establece una infección persistente (TB latente), situación que refleja un equilibrio biológico entre hospedero y patógeno, el cual es mantenido durante años por una red multifactorial $^{5}$.

La población chilena se formó por la mezcla entre amerindios de origen asiático, residentes del territorio nacional, y los inmigrantes españoles de origen caucásico que llegaron hace cinco siglos, con éstos también llegaron esclavos de origen africano. Los componentes ancestrales de la población mixta chilena actual han sido estudiados en base a marcadores genéticos informativos de ancestría, los que demostraron mayor proporción de componente amerindio en el norte y sur de Chile, mientras que el componente europeo es superior en Chile central ${ }^{6}$. En promedio, la contribución genética nativoamericana es de $(44,34 \% \pm 3,9 \%)$ y la europea de $(51,85 \% \pm 5,44 \%)$, es decir, son similares y significativamente más elevadas que el aporte africano $(3,81 \% \pm 0,45 \%)^{6,7}$. En la actualidad, los grupos amerindios más numerosos son la población Aymará, que se concentra en el norte del país (Arica, Parinacota), y la población Mapuche (en centro y sur del territorio). Por otra parte, estudios de ancestría espacial confirmaron que los chilenos son principalmente una mezcla de europeo y nativo-americano con un componente africano menor. Asimismo, que la población mixta chilena está claramente separada de la población africana, donde la ascendencia africana del suroeste de Estados Unidos resultó ser la más cercana. Ello, corroboraría la evidencia histórica acerca del origen oeste africano del linaje africano en las Américas. Asimismo, la proporción ancestral africana disminuye sistemáticamente de norte a sur en concordancia con la historia colonial local de la esclavitud en la costa del Pacífico de Sudamérica ${ }^{8}$.

El 2013 se reportó, que la incidencia de la TB en la población Aymará era 7 a 9 veces mayor 
que en la población no Aymará y la mortalidad se manifestaba a edad más temprana ${ }^{9}$. Asimismo, que la incidencia de la TB era consistentemente más alta en la población Mapuche, la que mostró 2,7 veces más riesgo a la TB que la población no Mapuche de la región del Bío-Bío ${ }^{10}$. El 2016, las mayores tasas de morbilidad total de TBTF ocurrieron en el norte de Chile (I: Tarapacá $[40,0 / 10.000 \mathrm{~h}]$; XV: Arica-Parinacota [26,7/100.000]); III: Atacama [16,1/100.000]); II: Antofagasta [15,7/100.000]). En la zona central la región Metropolitana presentó una tasa de [15,3/100.000] y la VIII región del Bíobío de [12,7/100.000]. En el sur la X región de Los Lagos [15,9/100.000]; la XIV de Los Ríos [13,5/100.000] y la IX de la Araucanía $[10,5 / 100.000])^{1}$. Coincidentemente el extremo norte de Chile, además de poseer un alto componente amerindio, tiene la ancestría africana más alta del país, así como en el sur lo es el componente Mapuche en la VIII y IX regiones, al igual que en la XIII' ${ }^{1}$.

Tanto, factores genéticos del ser humano ${ }^{11}$ como de los genotipos de $\mathrm{Mtb}^{12}$, contribuyen a la variabilidad interindividuo y de fenotipos de Mtb relacionados con la patogénesis y la presentación de la enfermedad ${ }^{12,13}$. La ecología de cepas prevalentes y su transmisión pueden variar dependiendo del continente, país o región. Chile, posee un aislamiento geográfico histórico, respecto de los países de Sudamérica, delimitado por la cordillera de Los Andes y el Océano Pacífico. Ello, se ha ido modificando en los últimos 10 años debido al ingreso de migrantes centro y suramericanos ${ }^{1}$.

Por otra parte, el estudio genotípico de Mtb, según técnicas de espoligotipo, MIRU-VNTR y RFLP, le ha categorizado en seis linajes genéticos (I aVI) las cepas circulantes de Mtb en el mundo: (I) Indo-oceánica (II); Asia Oriental (incluye Beijing); (III) África Oriental Indio; (IV) EuroAmericano; (V) África del Oeste (M. africanum); (VI) África del Oeste (M. africanum II), todas asociadas a regiones geográficas particulares ${ }^{11}$.

Recientemente se han descrito, en población sudafricana de color, ciertos genotipos predominantes de Mtb asociados específicamente a alelos HLA de clase I del hospedero. Por ejemplo, HLA-B27 se encuentra asociado a Mtb Beijing y múltiples episodios de TB pulmonar. Otras formas de TB se han asociado a cepas LAM $\left(\mathrm{A}^{*} 03\right)$, LCC $\left(B^{*} 07, B^{*} 44\right)$, Quebec $(B * 58)$ y linajes euroamericanos o del Este Asiático, evidencia que sustenta la hipótesis de la evolución conjunta del patógeno y ser humano ${ }^{11}$. Asimismo, se ha reportado que HLA DRB $1 * 15$ y DRB $1 * 08: 03$ se asocian con mayor incidencia de TB pulmonar en población de origen asiático ${ }^{14}$ y DRB $1 * 08: 03$, DQB1*06:01, DQB1*06:09 y DQA1*01:01 con mayores asociaciones genéticas en población caucásica que asiática. Además, a cierta población de India del sur, los alelos HLA DQB1*0601 y DR2 la predisponen a TB pulmonar ${ }^{15,16}$. Ningunos de los genotipos citados se han estudiado en la población chilena ni en cuanto a su relación con la susceptibilidad genética a la TB y/o al fenotipo de la enfermedad.

Recientemente, se demostró que la prevalencia de TB en pacientes con diabetes mellitus es 1,8 a 9,5 veces mayor en los países asiáticos en desarrollo que en la población general, hecho preocupante debido al incremento sostenido de la diabetes en la población mundial ${ }^{17}$. Ello, podría estar relacionado con lo descrito en una población afroamericana diabética tipo 1, donde HLADRB1*03, que incluye los alelos DRB1*03:01 y DRB1*03:02 (alelo escaso en europeos), confiere gran susceptibilidad o alta protección a diabetes tipo 1. Quizá, podría asociarse a la diversidad genética única de la región HLA africana, ausente en la población asiática ${ }^{18}$.

\section{Susceptibilidad genética a la tuberculosis}

Un polimorfismo genético corresponde a varias formas alternativas (alelos) para un mismo gen cuando tales variantes genéticas aparecen con una frecuencia $\geq 1 \%$ en la población. El tipo más común de polimorfismo es el de un solo nucleótido o SNP (Single Nucleotide Polymorphism). Un SNP es una forma de mutación puntual exitosa, desde el punto de vista evolutivo, que hace que ésta permanezca en una parte significativa de la población de una especie, contribuyendo a la diversidad genética.

En los últimos años se han revelado más de 30 polimorfismos genéticos implicados en susceptibilidad genética a la TB, éstos asociados a diferentes poblaciones y regiones geográficas del mundo. Probablemente, son consecuencia de miles de años de selección evolutiva patógenohospedero. El estudio de los factores genéticos humanos asociados a la TB pulmonar se ha centrado en la búsqueda de genes candidatos y recientemente en el análisis de la vinculación de todo el genoma basado en estudios de ligamiento en familias (family-based Genome-Wide Linkage Analyses $)^{19}$. Ambos, demuestran que la inmunidad innata es clave para el mecanismo de defensa primario del hospedero y consecuentemente de resistencia a la TB. Este fenómeno se asocia a variantes genéticas del hospedero, donde el mayor riesgo se asocia con los genes involu- 
crados en la respuesta inmune natural, tales como los receptores Toll-like (TLRs) presentes en los macrófagos; el receptor de vitamina D (VDR); NRAMP1 (SLC11A1; activación de macrófagos); INF- $\gamma$ y TNF- $\alpha$ (inmunodeficiencia); HLA I (B13-pulmonar tuberculosis) y HLA II (DR3 y DR7-protección relativa a TB); DR8-mayor riesgo a TB (inmunodeficiencia) ${ }^{20-23}$, entre otros.

Para comprender cómo las cepas de Mtb se adaptaron a las poblaciones humanas específicas, Salie et al., (2014) estudiaron los genes HLA clase I en pacientes de Ciudad del Cabo (Sudáfrica), la cual tiene una historia de 350 años de Mtb. Demostraron, que la cepa Beijing se aisla más frecuentemente de individuos con múltiples episodios de TB pulmonar y que el alelo HLAB*27 reduce el riesgo de contraer una cepa Beijing así como de episodios recurrentes de $\mathrm{TB}^{11}$.

Un alelo de NRAMP1 (activa al macrófago con Mtb intracelular), se asoció con mayor riesgo de TB pulmonar en una población de Gambia, África, Asia y Canadá, así como en niños de Texas, en su mayoría de origen hispano y africano, pero no en población de descendencia europea $^{22-26}$. La frecuencia del alelo [TGTGdel] de la región 3'UTR de NRAMP1, en una muestra de población China, fue de $20,4 \%$ en los enfermos de TB y de $11,1 \%$ en el grupo control sano ${ }^{27}$. Esta deleción tiene una frecuencia alélica de 0,13 en población mexicana, pero no se ha estudiado en otros países latinoamericanos ${ }^{28}$.

Los TLR (TLR 1, 2, 4, 6, 8 y 9) ${ }^{29,30}$, también presentes en las células dendríticas, reconocen ligandos de superficie de Mtb, provocando la activación de los genes involucrados en inflamación ${ }^{30}$. El TLR8 endosomal sensa el RNA bacteriano liberado dentro de la vacuola fagosomal, además "conversa" con otros TLR endosomales, permitiéndole modular la respuesta inmune a patógenos bacterianos, al inducir la expresión de NF- $\kappa \mathrm{B}$ e interferones ${ }^{31}$. Por ejemplo, la tribu Baiga, relativamente resistente a la TB pulmonar, expresa cantidades protectoras de IFN- $\gamma$ y TNF- $\alpha$, en comparación con las tribus Gond y Korku (todas de la Reserva Tigres Kanha, India). Esto, inhibe la replicación intracelular de Mtb y disminuye el riesgo de TB pulmonar ${ }^{23,32}$. De igual manera, en una población iraní, se describieron dos SNPs en TNF- $\alpha$, uno en posición -238 y otro en posición 857 , cuyos alelos A y $\mathrm{C}$, respectivamente, son más frecuentes en pacientes con TB pulmonar que en controles sanos. En el caso de INF- $\gamma$, el alelo $\mathrm{G}(+2109 \mathrm{~A} / \mathrm{G})$ resultó más frecuente en pacientes que en sujetos control, por ello se entiende que confiere mayor susceptibilidad a la $\mathrm{TB}^{33}$ a quienes lo portan. Asimismo,
Longhi et al., (2013) revisaron ciertos polimorfismos asociados a la respuesta inmune (Th1 o Th2) en poblaciones nativas de Brasil, Canadá, Irán, México, Noruega, Venezuela y Taiwán, donde la expresión de IFN- $\gamma$ y TNF- $\alpha$ siempre resultó menor en los sujetos nativos ${ }^{34}$. Yi et al., (2015) se refieren a los SNPs $-308 \mathrm{G}>\mathrm{A}$ y $-238 \mathrm{G}>\mathrm{A}$ del gen TNF- $\alpha$ asociados a susceptibilidad a la TB pulmonar, en africanos y asiáticos, respectivamente $^{35}$. En Chile, Santos et al., encontraron una frecuencia de 0,14 del alelo de riesgo en mujeres postmenopáusicas de Santiago ${ }^{36}$.

Recientemente, en Irán, se reportaron dos SNPs del gen TNF- $\alpha$ (-857 y -308) como prometedores marcadores para la identificación de población con riesgo a enfermar de $\mathrm{TB}^{37}$. También, se han descrito SNPs en TLR8 (cromosoma X), cuyos alelos menos frecuentes confieren susceptibilidad genética a la TB pulmonar en hombres de Indonesia y Rusia ${ }^{38-41}$, niños turcos hombres ${ }^{42}$, así como en cierta población pakistaní masculina $^{43}$. Dávila et al., (2008) describieron los SNPs Met1 Val (codón de inicio) y $-129 \mathrm{G} / \mathrm{C}$ (promotor gen), donde tanto el alelo 1 Val como el $\mathrm{C}$ se asociaron a mayor riesgo de $\mathrm{TB}^{40}$. La frecuencia de los alelos $1 \mathrm{Val}$ y $-129 \mathrm{G}$ se ha estudiado en varias etnias, demostrándose diferencias de susceptibilidad a la TB como de frecuencia entre población afroamericana, caucásica, japonesa y china ${ }^{44,45}$. No hay estudios en Chile de la frecuencia de estas variantes, pero en brasileros de ascendencia europea el alelo minoritario del codón de inicio tiene una frecuencia de 0,35 y en mujeres españolas sanas su frecuencia es $0,28^{46,47}$.

Los genes ADH1B y ALDH2, están involucrados en la metabolización de alcohol y acetaldehído. En una población coreana la frecuencia del alelo menor (ALDH2*487Lys) es más baja $(18,1 \%)$ en pacientes con TB que en el grupo control sano $(29,7 \%)$. En la población general la frecuencia de ALDH2*487Lys es de [0,161$0,181]$, excepto en alcohólicos [0,017] y en pacientes con TB $[0,099]$. Si la TB fue una enfermedad endémica ésta ejerció presión selectiva sobre el alelo ALDH2*487Lys en poblaciones asiáticas, lo que sugiere que este gen forma parte de uno de los componentes genéticos de la TB, al menos en la población coreana, japonesa y china investigada ${ }^{48}$. Es importante destacar que fuera del continente asiático la frecuencia de este alelo es muy escasa ${ }^{49}$.

El estudio de la concentración de vitamina D entre los contactos de pacientes con TB pulmonar en Castellón, España, demostró que una concentración de vitamina $\mathrm{D}[\geq 30 \mathrm{ng} / \mathrm{ml}]$ es suficiente para proteger a los contactos de la seroconver- 
sión determinada mediante test de tuberculina ${ }^{50}$. Previamente, se reportó en los Gujarati, una población vegetariana asiática, que la deficiencia de vitamina $\mathrm{D}$, se asociaba a TB activa, donde niveles indetectables conferían mayor riesgo ${ }^{51}$. El genotipo del receptor de vitamina D (VDR) también se asocia con susceptibilidad genética a la TB. Tal es el caso de VDR TaqI y VDR BsmI en la población Han Taiwanesa ${ }^{52}$. Asimismo, variantes de los polimorfismos de VDR (TaqI, BsmI y FokI) se asociaron con un riesgo significativamente mayor de desarrollar TB en Kazak (China) ${ }^{27}$, donde la frecuencia del alelo $\boldsymbol{f}$ fue de $43,7 \%$, en el grupo con TB y de $31,3 \%$ en los sujetos control y en población italiana sana se ha descrito una frecuencia de $37,5 \%$, no hay datos chilenos disponibles ${ }^{53}$.

Los genotipos homocigoto $(f f)$ y heterocigoto $(F f)$ confieren mayor riesgo en comparación con el genotipo homocigoto $(F F)$ WT (wild type) ${ }^{27}$. En sujetos europeos, el genotipo homocigoto $(b b)$ y el heterocigoto $(B b)$, del polimorfismo $B s m I$, se asociaron con un riesgo significativamente menor (efecto protector) de desarrollar TB en comparación con el homocigoto $(B B) \mathrm{WT}^{20}$.

La inmunidad antimicobacterias es heredable $\mathrm{y}$ un paciente con historia de TB pulmonar tiene más probabilidades de episodios recurrentes. En hermanos chilenos (gemelos/mellizos), vacunados con BCG, se demostró que si uno de ellos enfermó de TB, la probabilidad de que el otro la desarrolle es de $66,7 \%$ en gemelos monocigóticos y de solo $23 \%$ en mellizos dicigóticos ${ }^{54}$. En niños chilenos sanos la heredabilidad de la reactividad a tuberculina, después de la exposición a un caso de TB activa de adulto, se estimó en $92 \%$, sin correlación significativa entre niños no familiares que viven en el mismo hogar (estudio contacto $)^{55}$. De igual forma, en un estudio de contactos realizado en Colombia, la reactividad al test de tuberculina resultó dependiente del background genético de los sujetos ${ }^{56}$.

Recientemente, una investigación realizada en el Servicio de Salud Santiago Centro de Chile, demostró que la hipovitaminosis $\mathrm{D}$ era altamente prevalente en ciertos pacientes con TB así como en los respectivos contactos infectados de manera latente. Asimismo, una marcada estacionalidad, tanto para los niveles de vitamina D (25-hidroxicolecalciferol) como los contacto con infección latente, donde en invierno se encontró los niveles más reducidos de la vitamina y en primavera el riesgo más alto de TB latente. A su vez, los contactos de migrantes presentaron niveles de vitamina $\mathrm{D}$ más bajos que los no migrantes y tendencia a una mayor carga de infección latente ${ }^{57}$.
También se reveló una asociación inversa, estadísticamente significativa, entre la radiación solar y la incidencia de tuberculosis, resultados que sugieren un rol patogénico potencial de la vitamina $\mathrm{D}$ sobre la incidencia de la tuberculosis y su severidad ${ }^{58}$. Sin embargo, tales resultados no se asociaron a susceptibilidad genética a la TB, pero sí a mal nutrición y falta de exposición al sol.

La susceptibilidad mendeliana a enfermedad micobacteriana (MSMD) es una condición rara, observada en individuos sanos, caracterizada por la predisposición a desarrollar una enfermedad clínica a causa de micobacterias de baja virulencia, tales como la vacuna BCG y las micobacterias ambientales. Más aún, tal condición predispone a salmonellosis, candidiasis y tuberculosis y ocasionalmente a bacterias, hongos y parásitos intramacrofágicos y quizá a virus ${ }^{59}$. De hecho, la TB subcutánea, hematógena y extrapulmonar tienden a ser más frecuentes en infantes y adolescentes que en adultos, donde la TB primaria, potencialmente mortal en la infancia, se debería a inmunodeficiencias heredadas ${ }^{60}$. Se han descrito siete genes responsables de MSMD autosómicos (IFNGR1, IFNGR2, STAT1, IL12B, IL12RB1, ISG15, IRF8) y dos ligados al cromosoma $\mathrm{X}$ (NEMO, CYBB) vinculados con MSDS. Los nueve están fisiológicamente relacionados e involucrados en la inmunidad IFN- $\gamma$-dependiente. Esto, impide, por una parte, la producción de IL12B, IL12RB1, IRF8, ISG15, NEMO, y por el otro responder a IFN- $\gamma$ (IFNGR1, IFNGR2, STAT1, IRF8, CYBB). Las investigaciones realizadas en tales pacientes demostraron que la inmunidad mediada por IFN- $\gamma$ es esencial para el control de las infecciones micobacterianas y al parecer contra otros patógenos intramacrofágicos, virus y tumores. No obstante, existen pacientes con MSMD que no portan las mutaciones descritas lo que sugiere una gran heterogeneidad genética relacionada con esta patología ${ }^{59,60}$. De igual manera, la deficiencia de TYK-2 (autosómica recesiva), descrita en siete pacientes no familiares de distintas etnias, se relaciona con infecciones intracelulares por micobacterias (BCG, M. tuberculosis) y virus (VZV, HSV), debido a una respuesta celular disminuída a IL-12 e INF- $\alpha / \beta$, respectivamente, y al parecer también a bajos niveles de INF- $\gamma$. De los siete pacientes en estudio, cuatro, uno de Argentina, mostraron fenotipo MSMD o tuberculosis asociada a niveles deficientes de INF- $\gamma$; por ello debería considerarse como una deficiencia inmune primaria en casos de tuberculosis severa en niños ${ }^{61}$. 


\section{Genotipos de M. tuberculosis}

A pesar de los 5.000 años de convivencia de la humanidad con Mtb, éste posee alta homogeneidad genética con una divergencia de solo [5 $\mathrm{SNP} /$ genoma/año]. Los linajes de Mtb I, V y VI son considerados "antiguos"; II y IV "modernos", basados en la presencia o no de la región genómica TbD1, ausente en cepas modernas. Los linajes modernos se expandieron con más éxito como consecuencia del aumento de la población mundial ${ }^{44,45}$. Asimismo, algunos han emergido como dominantes en varios países y regiones geográficas, tales como en Sudáfrica (linajes II, III y IV); Chile, Estados Unidos, Argentina (linaje IV); África Oeste (linajes IV, V y VI) o Filipinas-Océano Índico (linaje I). También, el fenotipo de la enfermedad puede ser influenciado por el genotipo bacteriano. El linaje Beijing se asocia con mayor riesgo de transmisión, brotes y tuberculosis resistente a los medicamentos. El linaje euroamericano produce menos TB extrapulmonar, Beijing y $\mathrm{S}$ mayor riesgo de TB extrapulmonar e incluso se ha planteado que Beijing inhibe la liberación de TNF- $\alpha$ en macrófagos humanos THP-1 activados ${ }^{11}$.

En Chile, se investigó la diversidad genética de Mtb recolectados entre los años 2008 y 2013 en Santiago Centro. Los linajes relevantes resultaron ser los genotipos América Latina y Mediterráneo (LAM) (34\%) y T (33\%), seguidos de Haarlem $(16,5 \%)$. El 15,5\% correspondió a cepas huérfanas en la base de datos SITVIT2. La familia Beijing resultó con una baja representación $(1,9 \%)^{12}$. La familia SIT37/T3 mostró una alta proporción, a pesar que rara vez se encuentra en los países latinoamericanos; quizá ello revela una historia de Chile relacionada con migraciones humanas y evolución ${ }^{12,13}$.

\section{Conclusión}

Se requiere de estudios genéticos extensivos del hospedero para identificar subgrupos de individuos (edad de inicio, origen étnico, heredabilidad, polimorfismo, entre otros) y de factores ambientales (cepa del patógeno, nutrición, geografía, entre otros), que definan la vulnerabilidad intrínseca de cada individuo y de ciertas poblaciones de desarrollar TB, así como también acerca del genotipo y virulencia del Mtb infectante ${ }^{62-66}$.

En consecuencia, proponemos que en Chile existen pacientes con TB nueva y recurrente debido a su condición de portador de algún alelo de riesgo en SNPs ubicados en genes que confie- ren susceptibilidad genética a la $\mathrm{TB}$, semejante a lo descrito en otras poblaciones de América, Europa, África y Asia ${ }^{23,39,64,66}$. Obtener, tal evidencia científica permitiría considerar la variable genética al momento de diseñar los planes de prevención, control y erradicación de la $\mathrm{TB}$, en forma adicional a la mantención y refuerzo de las medidas del Programa Nacional de Control de la TB que han demostrado ser exitosas. El agregar el componente genético es relevante toda vez que en ausencia de una vacuna eficaz, nuestra mejor arma contra la TB continúa siendo nuestra inmunidad natural ${ }^{66}$.

\section{Bibliografía}

1.- Informe Mundial sobre la Tuberculosis 2017. Organización Mundial de la Salud. Disponible en: www.who.org. [Consultado el 30 de julio de 2018].

2.- HERRERA T. La tuberculosis en Chile: situación epidemiológica y avances del Programa Nacional de Control y Eliminación Rev Chil Enferm Respir 2017; 33: 320-4.

3.- Tuberculosis Informe Situación de Chile 2014. División Control y Prevención de Enfermedades. Junio 2015, Santiago, Chile. Disponible en: www.minsal.cl. [Consultado el 3 de enero de 2016].

4.- BRUNS H, STENGER S. New insights into the interaction of Mycobacterium tuberculosis and human macrophages. Future Microbiology 2014; 9 (3): 327-41. doi: $10.2217 / \mathrm{fmb} .13 .164$.

5.- DHEDA K, BARRY CE 3rd, MAARTENS G. Tuberculosis. Lancet 2016; 387: 1211-26. http://dx.doi. org/10.1016/S0140-6736(15)00151-8.

6.- CIFUENTES L. Proyecto Chilegenómico: Tras la diversidad genética de los chilenos. LII Boletín de la Academia Chilena de Medicina 2015; 13-36. Disponible en: http://www.institutodechile.cl/2012/noticias/portada/ boletin2015.pdf.

7.- FUENTES M, PULGAR I, GALLO C, BORTOLINI M-C, CANIZALES-QUINTERO S, BEDOYA G, et al. Geografía génica de Chile. Distribución regional de los aportes genéticos americanos, europeos y africanos. Rev Med Chile 2014; 142: 281-9.

8.- EYHERAMENDY S, MARTÍNEZ FI, MANEVY F, VIAL C, REPETTO G. Genetic structure characterization of chileans reflects historical immigration patterns. Nature communications 2015; doi: 10.1038/ ncomms 7472 .

9.- Situación de salud de la población Aymará. Indicadores básicos. Región de Arica y Parinacota. Secretaría Regional Ministerial de Salud. MINSAL, 2013. Chile. Disponible en: www.minsal.cl [Consultado el 2 de febrero de 2015].

10.- Secretaría Regional Ministerial de Salud. Diagnóstico de salud comunal de Alto Bío-Bío. MINSAL, 2013. Dispo- 
nible en: www.minsal.cl. [Consultado el 2 de febrero de 2015].

11.- SALIE M, VAN DER MERWE L, MÖLLER M, DAYA M, VAN DER SPUY GD, VAN HELDEN PD. et al. Associations Between Human Leukocyte Antigen Class I Variants and the Mycobacterium tuberculosis Subtypes Causing Disease JID 2014; 209: 216-23.

12.- BALCELLS ME, GARCÍA P, MEZA P, PEÑA C, CIFUENTES M, COUVIN D, et al. A first insight on the population structure of Mycobacterium tuberculosis complex as studied by spoligotyping and MIRU-VNTRs in Santiago, Chile. PLOS ONE 2015; 10 (2): e0118007. doi:10.1371/journal.pone.0118007.

13.- GAGNEUX S, SMALL PM. Global phylogeography of Mycobacterium tuberculosis and implications for tuberculosis product development. Lancet Infect Dis 2007; 7 (5): 328-37.

14.- LI CP, ZHOU Y, XIANG X, ZHOU Y, HE M. Relationship of HLA-DRB1 gene polymorphism with susceptibility to pulmonary tuberculosis: updated meta-analysis. Int J Tuberc Lung Dis 2015; 19 (7): 841-9. doi:10.5588/ ijtld.14.0521.

15.- OLIVEIRA-CORTEZ A, MELO AC, CHAVES VE, CONDINO-NETO A, CAMARGOS P. Do HLA class II genes protect against pulmonary tuberculosis? A systematic review and meta-analysis. Eur J Clin Microbiol Infect Dis 2016; 35 (10): 1567-80. doi: 10.1007/s10096016-2713-x.

16.- RAVIKUMAR M, DHEENADHAYALAN V, RAJARAM K, LAKSHMI SS, KUMARAN PP, PARAMASIVAN CN, et al. Associations of HLA-DRB1, DQB1 and DPB1 alleles with pulmonary tuberculosis in south India. Tuber Lung Dis 1999; 79 (5): 309-17.

17.- ZHENGA CH, HUA M, AND GAOB F. Diabetes and pulmonary tuberculosis: a global overview with special focus on the situation in Asian countries with high TBDM burden. Global Health Action 2017; 10: 1264702. doi.org/10.1080/16549716.2016.1264702.

18.- HOWSON JM, ROY MS, ZEITELS L, STEVENS H, TODD JA. HLA class II gene associations in African American type 1 diabetes reveal a protective HLADRB1*03 haplotype. Diabet Med 2013; 30 (6): 710-6.

19.- A Catalog of Published Genome-Wide Association Studies. National Human Genome Research Institute. Disponible en: http://www.ebi.ac.uk/gwas [Consultado el 10 de marzo de 2017].

20.- CHEN C, LIU Q, ZHU L, YANG H, LU W. Vitamin D Receptor Gene Polymorphisms on the Risk of Tuberculosis, a Meta-Analysis of 29 Case-Control Studies. PLoS ONE 2013; 8 (12). e83843. doi:10.1371/journal. pone. 0083843 .

21.- CHIMUSA ER, ZAITLEN N, DAYA M, MOLLER M, VAN HELDEN PD, MULDER NJ, et al. Genomewide association study of ancestry-specific TB risk in the South African Coloured population. Hum Mol Gen 2014; 23 (3): 796-809. doi:10.1093/hmg/ddt462.
22.- THADA S, VALLURI VL, GADDAM SL. Influence of Toll-Like Receptor Gene Polymorphisms to Tuberculosis Susceptibility in Humans. Scan J Immunol 2013; 78: 221-9.

23.- AZAD AK, SADEE W, SCHLESINGER LD. Innate Immune Gene Polymorphisms in Tuberculosis. Infect Immun 2012; 80: 3343. doi: 10.1128/IAI.00443-12.

24.- YOUNG BN, RENDO'N A, ROSAS-TARACO A, BAKER J, HEALY M, et al. The Effects of Socioeconomic Status, Clinical Factors, and Genetic Ancestry on Pulmonary Tuberculosis Disease in Northeastern Mexico. PLoS ONE 2014; 9(4): e94303. doi:10.1371/ journal.pone.0094303.

25.- DAVIES P, GRANGE JM. Factors affecting susceptibility and resistance to tuberculosis. Influence of vitamin $\mathrm{D}$ deficiency and vitamin D receptor polymorphisms on tuberculosis among Gujarati Asians in west London: a case-control study. Thorax 2001; 56 (Suppl II): ii23ii29P.

26.- MEYER GC, THYE T. Host genetic studies in adult pulmonary tuberculosis. Seminars in Immunology 2014; 26: 445-53. http://dx.doi.org/10.1016/j.smim. 2014.09.005.

27.- WU F, ZHANG W, ZHANG L, WU J, Li C, MENG X, et al. NRAMP1, VDR, HLA-DRB1, and HLA-DQB1 Gene Polymorphisms in Susceptibility to Tuberculosis among the Chinese Kazakh Population: A Case-Control Study. Biomed Res Int 2013; 2013: 484535. doi: 10.1155/2013/484535.

28.- HERNÁNDEZ-RIVERA MP, RAMÍREZ-RAMÍREZ A, CHIÑAS-PÉREZ A, MONROY-OSTRIA A, CANCINO-DÍAZ ME, HERNÁNDEZ-MONTES O. NRAMP1 Polymorphisms like Susceptibility Marker in Mexican Focus of Cutaneous Leishmaniasis. BioMed Research Int 2016; 7951285. doi:10.1155/2016/7951285.

29.- LAI Y, LIN TM, WANG CH, SU PY, WU JT, LIN MC, ENG HL. Functional polymorphisms of the TLR7 and TLR8 genes contribute to Mycobacterium tuberculosis infection. Tuberculosis 2016; 98: 125-131.

30.- SCHURZ H, DAYA M, MÖLLER M, HOAL EG, SALIE M. TLR1, 2, 4, 6 and 9 Variants Associated with Tuberculosis Susceptibility: A Systematic Review and Meta-Analysis. PLOS ONE 2015; 10 (10): e0139711. doi: 10.1371/journal.pone.0139711.

31.- CERVANTES JL, WEINERMAN B, BASOLE C, SALAZAR JC. TLR8: the forgotten relative revindicated. Cell Mol Immunol 2012; 1-5.

32.- BHARTI D, KUMAR A, MAHLA RS, KUMAR S, INGLE H, SHANKAR H, et al. The role of TLR9 polymorphism in susceptibility to pulmonary tuberculosis. Immunogenetics 2014; 66: 675-81. doi: 10.1007/ s00251-014-0806-1.

33.- VARAHRAM M, FARNIA P, NASIRI, MJ, KARAHRUDI MA, DIZAGIE MK, VELAYATI AA. Association of Mycobacterium Tuberculosis Lineages with IFN- $\gamma$ and TNF- $\alpha$ Gene Polymorphisms among 
Pulmonary Tuberculosis Patient. Mediterr J Hematol Infect Dis 2014; 6 (1): e2014015, DOI: 10.4084/ MJHID.2014.015.

34.- LONGHI RM, ZEMBRZUSKI VM, BASTA PC, CRODA J. Genetic polymorphism and immune response to tuberculosis in indigenous populations. Braz J Infect Dis 2013; 17 (3): 363-8. doi: 10.1016/j.bjid.2012.11.001.

35.- YI YX, HAN JB, ZHAO L, FANG Y, ZHANG YF, ZHOU GY. Tumor necrosis factor alpha gene polymorphism contributes to pulmonary tuberculosis susceptibility: evidence from a meta-analysis. Int J Clin Exp Med 2015; 8 (11): 20690-700.

36.- SANTOS JL, LERA L, PÉREZ-BRAVO F, ALBALA C. Adiposity and bone mineral density of Chilean elderly women in relation to toll-like receptor 4 gene polymorphisms. Ann Hum Biol 2006; 33 (5-6): 585-92.

37.- GHAMARI E, FARNIA P, SAIF S, MARASHIAN M, GHANAVI J, FARNIA P, et al. Comparison of single nucleotide polymorphisms [SNP] at TNF- $\alpha$ promoter region with TNF receptor 2 (TNFR2) in susceptibility to pulmonary tuberculosis; using PCR-RFLP technique. Am J Clin Exp Immunol 2016; 5 (4): 55-61.

38.- THYE T, VANNBERG FO, WONG SH, OWUSUDABO E, OSEI I, GYAPONG J, et al. Genome-wide association analyses identifies a susceptibility locus for tuberculosis on chromosome 18q11.2. Nat Genet 2010; 42 (9): 739-41. doi: 10.1038/ng.639.

39.- SALIE M, DAYA M, LUCAS LA, WARREN RM, VAN DER SPUY GD, et al. Association of toll-like receptors with susceptibility to tuberculosis suggests sex-specific effects of TLR8 polymorphisms. Infection, Genetics and Evolution 2015; 34: 221-9.

40.- DÁVILA S, HIBBERD ML, HARI DASS R, WONG HEE, SAHIRATMADJA E, BONNARD C, et al. Genetic Association and Expression Studies Indicate a Role of Toll-Like Receptor in Pulmonary Tuberculosis. PLOS Genetics 2008; 4 (10), e1000218. doi:10.1371/journal. pgen. 1000218.

41.- THADA S, VALLURI VL, GADDAM SL. Influence of Toll-like receptor gene polymorphisms to tuberculosis susceptibility in humans. Scand J Immunol 2013; 78 (3): 221-9. doi: 10.1111/sji.12066.

42.- DALGIC N, TEKIN D, KAYAALTI Z, CAKIR E, SOYLEMEZOGLU T, SANCAR M. Relationship between toll-like receptor 8 gene polymorphisms and pediatric pulmonary tuberculosis. Dis Markers 2011; 31 (1): 33-8. doi: 10.3233/DMA-2011-0800.

43.- BUKHARI M, ASLAM MA, KHAN A, IRAM Q, AKBAR A, NAZ AG, et al. TLR8 gene polymorphism and association in bacterial load in southern Punjab of Pakistan: an association study with pulmonary tuberculosis. Int J Immunogenetics 2015; 42: 46-51. doi:10.1111/iji.12170.

44.- GAGNEUX S. Host-pathogen coevolution in human tuberculosis. Phil Trans R Soc B 2012; 367 (1590): 850-9.
45.- BELAY M, AMENI G, BJUNE G, COUVIN D, RASTOGI N, AND ABEBE F. Strain Diversity of Mycobacterium tuberculosis Isolates from Pulmonary Tuberculosis Patients in Afar Pastoral Region of Ethiopia. BioMed Res Int 2014; 2014: 238532. doi: $10.1155 / 2014 / 238532$.

46.- VALVERDE-VILLEGAS JM, DOS SANTOS BP, DE MEDEIROS RM, MATTEVI VS, LAZZARETTI RK, SPRINZ E, et al. Endosomal toll-like receptor gene polymorphisms and susceptibility to HIV and HCV co-infection-Differential influence in individuals with distinct ethnic background. Hum Immunol 2017; 78 (2): 221-6. doi:10.1016/j.humimm.2017.01.001.

47.- MARTÍNEZ-ROBLES E, YEBRA-BANGO M, MELLOR-PITA S, TUTOR-URETA P, VARGAS JA, CITORES MJ. Genotypic distribution of common variants of endosomal toll like receptors in healthy Spanish women. A comparative study with other populations. Gene 2016; 578 (1): 32-7. doi:10.1016/j.gene.2015.12.004.

48.- PARK BSK, PARK CS, LEE HS, PARK KS, PARK BL, CHEONG HS, et al. Functional polymorphism in aldehyde dehydrogenase-2 gene associated with risk of Tuberculosis. BMC Med Gen 2014; 15:40. https://doi. org/10.1186/1471-2350-15-40. http://www.biomedcentral.com/1471-2350/15/40.

49.- GOEDDE HW, AGARWAL DP, FRITZE G, MEIERTACKMANN D, SINGH S, BECKMANN G, et al. Distribution of $\mathrm{ADH} 2$ and $\mathrm{ALDH} 2$ genotypes in different populations. Hum Genet 1992; 88 (3): 344-6.

50.- ARNEDO-PENA A, JUAN-CERDÁN JV, ROMEUGARCÍA A, GARCÍA-FERRER D, HOLGUÍNGÓMEZ R, IBORRA-MILLET J, et al. Vitamin D status and incidence of tuberculosis among contacts of pulmonary tuberculosis patients. Int J Tuberc Lung Dis 2015; 19 (1): 65-69 (5).

51.- MARTINEAU AR, LEANDRO AC, ANDERSON ST, NEWTON SM, WILKINSON KA, NICOL MP, et al. Association between Gc genotype and susceptibility to TB is dependent on vitamin D status. Eur Respir J 2000; 35 (5): 1106-12. doi: 10.1183/09031936.00087009.

52.- LEE S-W, CHUANG T-Y, HUANG H-H, LIU C-W, KAO Y-H, WU L S-H. VDR and VDBP genes polymorphisms associated with susceptibility to tuberculosis in a Han Taiwanese population. J Microbiol Immunol Inf 2016; 49: 783-7. doi: http://dx.doi.org/10.1016/j. jmii.2015.12.008.

53.- COLOMBINI A, BRAYDA-BRUNO M, LOMBARDI G, CROISET SJ, VRECH V, MAIONE V, et al. FokI polymorphism in the vitamin $\mathrm{D}$ receptor gene (VDR) and its association with lumbar spine pathologies in the Italian population: a case-control study. PLOS ONE 2014; 8;9 (5): e97027. doi:10.1371/journal. pone. 0097027.

54.- SEPÚLVEDA RL, HEIBA IM, NAVARRETE C, ELSTON RC, GONZÁLEZ B, SORENSEN RU. Tuberculin reactivity after newborn BCG immunization in mono- 
and dizygotic twins. Tuber Lung Dis 1994; 75 (2): 13843.

55.- SEPÚLVEDA RL, HEIBA IM, KING A, GONZÁLEZ B, ELSTON RC, SORENSEN RU. Evaluation of tuberculin reactivity in BCG-immunized siblings. Am J Respir Crit Care Med 1994; 149 (3 Pt 1): 620-4.

56.- COBAT A, BARRERA LF, HENAO H, ARBELÁEZ P, ABEL L, GARCÍA LF, et al. Tuberculin skin test reactivity is dependent on host genetic background in Colombian tuberculosis household contacts. Clin Infect Dis 2012; 54: 968-71. doi: 10.1093/cid/cir972.

57.- BALCELLS ME, GARCÍA P, TIZNADO C, VILLARROEL L, SCIOSCIA N, CARVAJAL C, et al. Association of vitamin D deficiency, season of the year, and latent tuberculosis infection among household contacts. PLOS ONE 2017; 12 (4): e0175400. https:// doi. org/10.1371/journal.pone.0175400.

58.- BALCELLS ME, CERDA J, CONCHA S, HOYOSBACHILOGLU R, CAMARGO CA, MARTINEAU $\mathrm{AR}$, et al. Regional solar radiation is inversely correlated with incidence and severity of tuberculosis in Chile. Epidemiol Infect 2017; 145: 1815-23. doi: 10.1017/ S0950268817000607.

59.- BUSTAMANTE J, BOISSON-DUPUIS S, ABEL L, CASANOVA J-L. Mendelian susceptibility to mycobacterial disease: genetic, immunological, and clinical features of inborn errors of IFN- $\gamma$ immunity. Seminars Immunology 2014; 26 (6): 454-70.

60.- BOISSON-DUPUIS S, BUSTAMANTE J, ELBAGHDADI $J$, et al. Inherited and acquired immu- nodeficiencies underlying tuberculosis in childhood. Immunological Rew 2015; 264: 103-20. doi:10.1111/ imr.12272.

61.- KREINS AY, CIANCANELLI MJ, OKADA S, KONG XF, RAMÍREZ-ALEJO N, et al. Human TYK2 deficiency: Mycobacterial and viral infections without hyper-IgE síndrome. J Exp Med 2015; 212: 1641-62 www.jem.org/cgi/doi/10.1084/jem.20140280.

62.- FERNÁNDEZ-MESTRE M, VILLASMIL A, TAKIFF H, FUENTES Z. NRAMP1 and VDR Gene Polymorphisms in Susceptibility to Tuberculosis in Venezuelan Population. Disease Markers 2015; ID 860628: http:// dx.doi.org/10.1155/2015/860628.

63.- KIM L, MOONAN PK, YELK RS, KAMMERER JS, HADDAD MB. Epidemiology of recurrent tuberculosis in the United States, 1993-2010. Int J Tuberc Lung Dis 2013; 17: 357-60. doi:10.5588/ijtld.12.0640.

64.- MEYER CG, THYE T. Host genetic studies in adult pulmonary tuberculosis. Sem Immunol 2014; 26:44553.

65.- WALLIS RS, KIM P, COLE S, HANNA D, ANDRADE BB, MAEURER M, et al. Tuberculosis biomarkers discovery: developments, needs, and challenges. Lancet Infect Dis 2013; 13: 362-72.

66.- YOUNG BN, RENDÓN A, ROSAS-TARACO A, BAKER J, HEALY M, GROSS JM, et al. The Effects of Socioeconomic Status, Clinical Factors, and Genetic Ancestry on Pulmonary Tuberculosis Disease in Northeastern Mexico. PLOS ONE 2014; 9: e94303. doi: 10.1371/journal.pone.0094303.
Correspondencia a:

Nancy P. Maulén L.

Escuela de Nutrición y Dietética. Facultad de Medicina.

Universidad de Los Andes. Santiago, Chile.

Email:n.maulen@gmail.com 